\title{
Predictive Ansatz for Fermion Masses in SUSY GUTS
}

\author{
G. K. Leontaris \\ Theoretical Physics Division \\ University of Ioannina \\ GR-451 10 Ioannina \\ Greece
}

\author{
N. D. Tracas \\ Physics Department \\ National Technical University \\ GR-157 80 Zografou \\ Athens, Greece
}

\begin{abstract}
We reexamine a succesful fermion mass Ansatz proposed by Giudice for a wide range of the ratio $\tan \beta=\frac{<\bar{h}>}{\langle h\rangle}$ (where $\bar{h}, h$ are the two standard higgs fields), in the context of supersymmetric grand unified theories. We find that the 7 predictions of the ansatz, $V_{u s}, V_{c b}, V_{u b}, m_{u}, m_{d}, m_{s}$ and $m_{b}$ are in good agreement with the experiment when either $i$ ) $\tan \beta \simeq 1$ or ii) $\tan \beta \geq 30$. A correct prediction for the bottom mass gives a lower limit on $m_{t} \geq 125$ for case $(i)$, in agreement with the previous analysis, while in case (ii) $m_{t} \geq 145$.
\end{abstract}

IOA $281 / 92$

NTUA $37 / 92$

December 1992 
There is a firm belief among the high energy physicists that in the ultimate theory, all the arbitrary parameters of the standard model will be determined only from a small number of inputs at some unification scale.

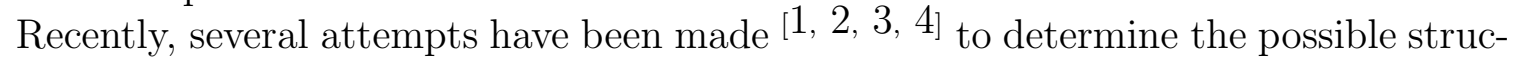
tures of the fermion mass matrices at the Grand Unification(GUT) scale, which lead to the correct low energy mass spectrum and to the maximal number of predictions. In Ref.[2], a simple ansatz for the fermion mass matrices, at the GUT scale, was proposed. The 13 arbitrary parameters of the low energy were determined by 6 inputs, hence leading to 7 predictions.

In a recent paper [5, the original ansatz of Ref. [2] for the fermion mass matrices was expanded in order to incorporate non-zero neutrino masses, as these are naturally predicted in most of the GUT models. With only 2 additional inputs at the GUT scale, it was found that all the previous succesful predictions are retained, while one gets seven new low energy predictions: the masses of the 3 light neutrinos, the leptonic mixing angles and the corresponding CP-phase. It was also proposed that the $m_{\nu_{\tau}}$ mass can give the correct contribution to the hot dark matter, in agreement with the interpretation of the COBE data 6, while the solar neutrino problem is solved via the MSW-mechanism 8 and $\nu_{\mu} \rightarrow \nu_{e}$ oscillations 17 .

All the previous calculations however, have been done for the case of small bottom Yukawa coupling, compared to that of the top quark. This corresponds to a relatively small ratio $\tan \beta$ of the two Higgs vev's $\langle\bar{h}\rangle$ and $\langle h\rangle$ which give masses to the up and down quarks respectively. In this particular case one can ignore all but the top Yukawa coupling corrections in the renormalization group equations (RGEs) of the Yukawa couplings, and calculate to a good approximation all the low energy parameters from the inputs at the GUT scale.

In many unified models however - and in particular in string derived GUTs - it is quite possible for the top and bottom quark Yukawa couplings to be comparable at the GUT scale. In that case the bottom coupling corrections play also an important rôle and should not be ignored. Such a case would obviously correspond to a pretty large value of $\tan \beta$, in contrast to the previous case.

In the present letter we are going to explore this latter case. We start with an overview of the basic features of the proposed framework 21. It is assumed that there exists some Grand Unified Supersymmetric Model (i.e. SO(10), SU(5), SU(4) etc) with the following form of the mass matrices at the GUT scale

$$
\begin{aligned}
M_{u}= & \left(\begin{array}{lll}
0 & 0 & b \\
0 & b & 0 \\
b & 0 & a
\end{array}\right), \quad M_{\nu \nu^{c}}=\left(\begin{array}{ccc}
0 & 0 & b \\
0 & b & 0 \\
b & 0 & a
\end{array}\right), \\
M_{d}= & \left(\begin{array}{ccc}
0 & f e^{i \phi} & 0 \\
f e^{-i \phi} & d & 2 d \\
0 & 2 d & c
\end{array}\right), \quad M_{e}=\left(\begin{array}{ccc}
0 & f e^{i \phi} & 0 \\
f e^{-i \phi} & -3 d & 2 d \\
0 & 2 d & c
\end{array}\right), \\
& M_{\nu^{c} \nu^{c}}=M \operatorname{diag}\left(k^{-2}, k^{-1}, 1\right) .
\end{aligned}
$$

There is a factor of -3 difference in the $\{22\}$ entry of the matrix $M_{e}$ compared to that of $M_{d}$. This arises naturally whenever these entries are coupled to Higgs doublets belonging to specific representations of the GUT group (또 of $\mathrm{SU}(5)$ or $\underline{126}$ of $\mathrm{SO}(10)$ ). 
The relation of the $\{22\}$ and $\{23\}$ entries in $M_{d}, M_{e}$ matrices is just a phenomenological assumption 21. The original ansatz for the $M_{u}, M_{d}$ and $M_{e}$ matrices was augmented by a simple assumption for the Dirac, $M_{\nu \nu^{c}}$, and the heavy Majorana, $M_{\nu^{c} \nu^{c}}$, neutrinos mass matrices: $M_{\nu \nu^{c}}$ is simply taken to be identical to $M_{u}$, due to the GUT relations, while $M_{\nu^{c} \nu^{c}}$ is taken for simplicity diagonal, whose elements differ by a hierarchy factor $k \approx 10$. The predictions in the neutrino sector have been discussed elsewhere [5], thus we are not going to elaborate them here.

The RGE's for the Yukawa couplings at the one loop level are

$$
\begin{aligned}
16 \pi^{2} \frac{d}{d t} \lambda_{U} & =\left(I \cdot \operatorname{Tr}\left[3 \lambda_{U} \lambda_{U}^{\dagger}\right]+3 \lambda_{U} \lambda_{U}^{\dagger}+\lambda_{D} \lambda_{D}^{\dagger}-I \cdot G_{U}\right) \lambda_{U} \\
16 \pi^{2} \frac{d}{d t} \lambda_{N} & =\left(I \cdot \operatorname{Tr}\left[\lambda_{U} \lambda_{U}^{\dagger}\right]+\lambda_{E} \lambda_{E}^{\dagger}-I \cdot G_{N}\right) \lambda_{N} \\
16 \pi^{2} \frac{d}{d t} \lambda_{D} & =\left(I \cdot \operatorname{Tr}\left[3 \lambda_{D} \lambda_{D}^{\dagger}+\lambda_{E} \lambda_{E}^{\dagger}\right]+3 \lambda_{D} \lambda_{D}^{\dagger}+\lambda_{U} \lambda_{U}^{\dagger}-I \cdot G_{D}\right) \lambda_{D}, \\
16 \pi^{2} \frac{d}{d t} \lambda_{E} & =\left(I \cdot \operatorname{Tr}\left[\lambda_{E} \lambda_{E}^{\dagger}+3 \lambda_{D} \lambda_{D}^{\dagger}\right]+3 \lambda_{E} \lambda_{E}^{\dagger}-I \cdot G_{E}\right) \lambda_{E},
\end{aligned}
$$

where $\lambda_{\alpha}, \alpha=U, N, D, E$, represent the 3x3 Yukawa matrices which are defined in terms of the mass matrices given in Eq.(11-3), and $I$ is the $3 \times 3$ identity matrix and

$$
\begin{aligned}
G_{\alpha} & =\sum_{i=1}^{3} c_{\alpha}^{i} g_{i}^{2}(t), \\
g_{i}^{2}(t) & =\frac{g_{i}^{2}\left(t_{0}\right)}{1-\frac{b_{i}}{8 \pi^{2}} g_{i}^{2}\left(t_{0}\right)\left(t-t_{0}\right)} \\
\left\{c_{U}^{i}\right\}_{i=1,2,3} & =\left\{\frac{13}{15}, 3, \frac{16}{3}\right\}, \quad\left\{c_{D}^{i}\right\}_{i=1,2,3}=\left\{\frac{7}{15}, 3, \frac{16}{3}\right\}, \\
\left\{c_{E}^{i}\right\}_{i=1,2,3} & =\left\{\frac{9}{5}, 3,0\right\}, \quad\left\{c_{N}^{i}\right\}_{i=1,2,3}=\left\{\frac{3}{5}, 3,0\right\} .
\end{aligned}
$$

Following Ref.[2] we diagonalize the up quark Yukawa matrix at the GUT scale and redefine properly the lepton and quark fields

$$
\begin{array}{rlrl}
\lambda_{U} \rightarrow \tilde{\lambda}_{U} & =K^{\dagger} \lambda_{U} K, & & \lambda_{N} \rightarrow \tilde{\lambda}_{N}=K^{\dagger} \lambda_{N} K, \\
\lambda_{D} \rightarrow \tilde{\lambda}_{D}=K^{\dagger} \lambda_{D} K, & \lambda_{E} \rightarrow \tilde{\lambda}_{E}=K^{\dagger} \lambda_{E} K .
\end{array}
$$

where now $\tilde{\lambda}_{U}$ is diagonal and $K$ is

$$
K=\left(\begin{array}{ccc}
\cos \theta & 0 & \sin \theta \\
0 & 1 & 0 \\
-\sin \theta & 0 & \cos \theta
\end{array}\right), \quad \tan 2 \theta=\frac{2 b}{a} .
$$

Now assuming that the only significant Yukawa terms are $\tilde{\lambda}_{U_{33}}, \tilde{\lambda}_{D_{33}}$ and $\tilde{\lambda}_{E_{33}}$, to a good approximation we may drop all other terms in the parentheses of the right-handed side of the RGE's above, and write them formally as follows

$$
\tilde{\lambda}_{U}(t)=\gamma_{U}\left(\begin{array}{ccc}
1 & 0 & 0 \\
0 & 1 & 0 \\
0 & 0 & \zeta \xi^{3}
\end{array}\right) \xi^{3} \tilde{\lambda}_{U}\left(t_{0}\right)
$$




$$
\begin{aligned}
& \tilde{\lambda}_{D}(t)=\gamma_{D}\left(\begin{array}{ccc}
1 & 0 & 0 \\
0 & 1 & 0 \\
0 & 0 & \xi \zeta^{3}
\end{array}\right) \zeta^{3} \zeta^{\prime} \tilde{\lambda}_{D}\left(t_{0}\right) \\
& \tilde{\lambda}_{E}(t)=\gamma_{E}\left(\begin{array}{ccc}
1 & 0 & 0 \\
0 & 1 & 0 \\
0 & 0 & \zeta^{\prime 3}
\end{array}\right) \zeta^{3} \zeta^{\prime} \tilde{\lambda}_{E}\left(t_{0}\right) \\
& \tilde{\lambda}_{N}(t)=\gamma_{N}\left(\begin{array}{ccc}
1 & 0 & 0 \\
0 & 1 & 0 \\
0 & 0 & \zeta^{\prime}
\end{array}\right) \xi \tilde{\lambda}_{N}\left(t_{0}\right)
\end{aligned}
$$

where

$$
\begin{aligned}
\gamma_{\alpha}(t) & =\exp \left(-\int G_{\alpha}(t) d t /\left(16 \pi^{2}\right)\right) \\
\xi & =\exp \left(\frac{1}{16 \pi^{2}} \int_{t_{0}}^{t} \tilde{\lambda}_{t} d t\right) \\
\zeta & =\exp \left(\frac{1}{16 \pi^{2}} \int_{t_{0}}^{t} \tilde{\lambda}_{b} d t\right) \\
\zeta^{\prime} & =\exp \left(\frac{1}{16 \pi^{2}} \int_{t_{0}}^{t} \tilde{\lambda}_{\tau} d t\right)
\end{aligned}
$$

where $\tilde{\lambda}_{t}, \tilde{\lambda}_{b}$ and $\tilde{\lambda}_{\tau}$ stand for $\tilde{\lambda}_{U_{33}}, \tilde{\lambda}_{D_{33}}$ and $\tilde{\lambda}_{E_{33}}$ respectively. In the above equations $\tilde{\lambda}_{\tau}$ is also included since it satisfies the same initial condition with $\tilde{\lambda}_{b}$. Of course the evolution down to $M_{Z}$ is different and $\zeta^{\prime}$ stays very close to 1 as $\tilde{\sim}_{\tilde{\lambda}} \tilde{\lambda}_{\tau}$ as the initial value of $\tilde{\lambda}_{\tau}$ does not get too large. Note that in the limit where $\tilde{\lambda}_{t} \gg \tilde{\lambda}_{b}, \tilde{\lambda}_{\tau}$ we get $\zeta \approx \zeta^{\prime} \approx 1$ and the above equations reduce to the simple uncoupled form

$$
\tilde{\lambda}_{\alpha}(t)=\xi^{k} \gamma_{\alpha} \lambda_{\alpha}\left(t_{0}\right)
$$

where now

$$
\xi=\left(1-\frac{k}{8 \pi^{2}} \tilde{\lambda}_{t}\left(t_{0}\right) \int_{t_{0}}^{t} \gamma_{\alpha}^{2}(t) d t\right)^{\left(\frac{-1}{2 k}\right)}
$$

and we recover the previous results 2 . 5 . In the general case, however, the differential equations remain coupled and only a numerical solution is possible.

We obtain the following relations among the masses

$$
m_{t}=\zeta \xi^{3} \frac{\eta_{u} m_{c}^{2}}{\eta_{c}^{2} m_{u}}
$$

and for the down quarks and leptons

$$
\begin{aligned}
& m_{b} \approx \frac{\gamma_{D}}{\gamma_{E}} \frac{\zeta^{3}}{\zeta^{\prime 3}} \xi m_{\tau} \eta_{b} \\
& m_{s} \approx \eta_{s} \frac{\gamma_{D}}{\gamma_{E}} \frac{m_{\mu}}{3}\left(1-\frac{4}{9}\left(1+3 \zeta^{\prime 3}\right) \frac{m_{\mu}}{m_{\tau}}\right) \\
& m_{d} \approx \eta_{d} \frac{\gamma_{D}}{\gamma_{E}} 3 m_{e}\left(1+\frac{4}{9}\left(1+3 \zeta^{\prime 3}\right) \frac{m_{\mu}}{m_{\tau}}\right)
\end{aligned}
$$


where in the above relations $\eta_{\alpha},(\alpha=b, c)$ are taking into account the QCD renormalization effects of the corresponding quark masses from the energy scale $m_{t}$ down to their masses $\eta_{\alpha}=m_{\alpha}\left(m_{\alpha}\right) / m_{\alpha}\left(m_{t}\right)$, while for $(\alpha=u, d, s)$ we use $\eta_{\alpha}=m_{\alpha}(1 \mathrm{GeV}) / m_{\alpha}\left(m_{t}\right)$. In what follows, we use the values $\eta_{b}=1.4, \eta_{c}=1.8$ and $\eta_{u}=\eta_{d}=\eta_{s}=2.0$. The bottom quark mass is taken to lie in the range $m_{b}\left(m_{b}\right)=4.25 \pm .1 \mathrm{GeV}$. Thus, from the relation (26), the correct prediction for $m_{b}$, fixes the combination $\xi\left(\zeta / \zeta^{\prime}\right)^{3} \approx .81 \pm .02$ (the rest of the renormalization group parameters involved in (26) vary slowly in terms of the input parameters). In order to expess the predictions for the Kobayashi Maskawa (KM) mixing angles, we use the following parametrization for the KM-matrix

$$
V_{K M}=\left(\begin{array}{ccc}
c_{1} c_{3} e^{i \phi}-s_{1} s_{2} s_{3} & s_{1} c_{3} e^{i \phi}+c_{1} s_{2} s_{3} & -c_{2} s_{3} \\
-s_{1} c_{2} & c_{1} c_{2} & s_{2} \\
c_{1} s_{3} e^{i \phi}+s_{1} s_{2} c_{3} & s_{1} s_{3} e^{i \phi}-c_{1} s_{2} c_{3} & c_{2} c_{3}
\end{array}\right)
$$

where $c_{1}=\cos \theta_{1}, s_{1}=\sin \theta_{1}$, etc. Then the predictions are:

$$
\begin{aligned}
V_{u s} & \simeq 3: s_{1}: \simeq \sqrt{\frac{m_{e}}{m_{\mu}}}\left(1-\frac{25}{2} \frac{m_{e}}{m_{\mu}}+\frac{16}{9} \frac{m_{\mu}}{m_{\tau}}\right) \\
V_{c b} & \simeq: s_{2}: \simeq \frac{2}{3}\left(\xi \zeta^{3}\right)^{-1} \frac{m_{\mu}}{m_{\tau}}\left(1-\frac{m_{e}}{m_{\mu}}-\frac{1}{9} \frac{m_{\mu}}{m_{\tau}}\right), \\
V_{u b} & \simeq: s_{3}: \simeq\left(\frac{\xi}{\zeta}\right)^{2} \frac{m_{c}}{\eta_{c} m_{t}} .
\end{aligned}
$$

In order to compute the various renormalization group parameters which enter the various relations given above, we solve numerically the equations (14-16) assuming the initial condition at $M_{G U T} \simeq 10^{16} \mathrm{GeV}$, with $g_{G U T} \simeq \frac{1}{25.1}$. We are taking supersymmetric beta function coefficients from $M_{G U T}$ down to $m_{t}$, while below $m_{t}$ we run the system with non-supersymmetric ones. We ensure that the gauge couplings lie in the experimentally accepted region at $m_{W}$ and we compute the quark masses for a wide range of $\tan \beta$, each time using the proper initial values for the couplings $\lambda_{0, t}, \lambda_{0, b}$. Obviously, when $\tan \beta \simeq 1$, a small ratio $r=\frac{\lambda_{0, b}}{\lambda_{0, t}}$ is needed, while when $r \simeq 1$ then $\tan \beta \gg 1$. Note however, that $\lambda_{0, b}=\lambda_{0, \tau}$, while $\lambda_{0, \tau}$ and $\tan \beta$ should also be chosen so as to give the correct $\tau$ mass. Thus, a consistency check is done for each chosen pair of values $\left(\lambda_{0, \tau}, \tan \beta\right)$ seperately, where the $\tau$ mass is taken to be $m_{\tau}=1784.1 \mathrm{MeV}$. Our numerical analysis reproduces the previous results when $\tan \beta \leq 5$ and extends the analysis to the case where $\tan \beta \gg 1$.

In order to see clearly the effect of a large $\tan \beta$, (or equivallently a large $\lambda_{0, b}$ coupling), in figure (1a) we plot the bottom mass versus the bottom coupling $\lambda_{0, b}$ at the GUT scale, for constant top-mass $m_{t}=145 \mathrm{GeV}$, for three successive approximations: Contour (I) represents the case where $\lambda_{0, b}, \lambda_{0, \tau}$-corrections are neglected. Contour (II) represents the solution where only $\lambda_{0, \tau}$ correction is neglected, while case (III) is the contour which corresponds to the complete differential system (14-16) where the corrections from all three couplings are taken into account in the running. All curves are almost identical for small $\lambda_{b}$ and $\tan \beta<5$. Curve (I) is no longer valid for $\tan \beta>5$ while curve (II) is not a good approximation for $\tan \beta>10$. Case (III), but in terms of the ratio $r=\frac{\lambda_{0, b}}{\lambda_{0, t}}$ is shown in figure (1b). However, for this particular value of $m_{t}$, reasonably large values of $\tan \beta(\sim 10)$, are excluded from the bottom mass range which is shown as the shaded region of these figures. Acceptable $m_{b}$ values are possible only for $\tan \beta \geq 40($ !), but this 
corresponds to the unlikely case of $r>1$. Moreover, additional constraints arise from the KM-mixing angles which are given in (29-32). In particular, for the $m_{t}=145 \mathrm{GeV}$ case, the element $V_{b c}$ is in its upper experimentally allowed limit $\left(\simeq 5.4 \times 10^{-2}\right)$, only when $r \equiv \frac{\lambda_{0, b}}{\lambda_{0, t}} \simeq 1$, while - unless $r \ll 1$ - all the rest of the region of $r$ is excluded either from $m_{b}$ or from $V_{b c}$ constraints. In fact, the value $m_{t} \simeq 145 \mathrm{GeV}$, is the lower top mass which can be obtained for a large $\lambda_{b}$ coupling, while smaller top mass values can be obtained only for a neglegible $\lambda_{b}$ coupling. In table I we collect various results for the experimentally measured parameters for various values of $m_{t}$. For all these cases we find that $m_{d} \approx 6.8 \mathrm{MeV}$ and $m_{s} \approx 138 \mathrm{MeV}$.

As $m_{t}$ gets higher however, smaller $\tan \beta$ values are possible. In figure (2) we show such a case for $m_{t}=170 \mathrm{GeV}$ where we plot $m_{b}$ versus $\tan \beta$. A comparison of the two curves in terms of the $\lambda_{b}$ values is presented in figure (3). The shaded area (whose upper bound corresponds to $\left.m_{t} \simeq(170 \pm 3) G e V\right)$, is prevented due to the bad ratio $\frac{m_{u}}{m_{c}}$. Thus, it is remarkable that this ratio which is derived only in terms of the running $m_{u}, m_{c}$ masses determined by well known methods 9 , can put an upper bound on the top-quark mass. If, on the other hand, from the electroweak breaking mechanism in supesymmetric models we demand the condition $\tan \beta>1$, then we obtain a lower bound $m_{t} \geq 125 \mathrm{GeV}$, although, this second bound is reffered to a very small region as can be seen from figure (4).

In conclusion, we have reconsidered a proposed 2 ansatz for the fermion mass matrices at the GUT scale, and studied in detail the effects of a large bottom Yukawa coupling on the various experimentally measured parameters of the low energy theory. We have shown that the renormalization corrections of the $\lambda_{b}$ Yukawa coupling have a significant impact whenever $r=\frac{\lambda_{0, b}}{\lambda_{0, t}} \geq 0.1$, while when $r \ll 1$, they can safely be neglected. Furthermore, for $r \geq 1, m_{b^{-}}$and $V_{c b^{-}}$low energy bounds put a lower limit on the top mass $m_{t} \geq 145 \mathrm{GeV}$, while in the case of $r \ll 1$, one gets a less restrictive top mass $m_{t} \geq 125 \mathrm{GeV}$. In both cases, an upper bound on the top mass can be obtained, $m_{t} \leq 173 \mathrm{GeV}$, from the relation (25).

The work of G.K.L. is partially supported by a C.E.C Science Program SCI-0221C(TT), while of N.D.T by C.E.C. Science Program SC1-CT91-0729. 


\section{TABLE I.}

The predictions for the Ansatz of ref[2] for large values of $\lambda_{0, b}$ and $\tan \beta$. The corresponding experimental ranges are $V_{u b}=(4 \pm 3) \times 10^{-3}, V_{c b}=(4.3 \pm 1.1) \times 10^{-2}$.10 From (27-30) we also get $m_{d} \approx 6.8 \mathrm{MeV}, m_{s} \approx 138 \mathrm{MeV}$ and $V_{u b} \approx .218$, in agreement with the experiment.

\begin{tabular}{||c|c|c|c|c|c|c||}
\hline \hline$m_{t}(\mathrm{GeV})$ & $r$ & $V_{u b} \times 10^{3}$ & $V_{c b} \times 10^{2}$ & $m_{b}(\mathrm{GeV})$ & $\tan \beta$ & \\
\hline \hline \multirow{3}{*}{145} & $\sim 1.1$ & $4.90 \pm 0.20$ & 5.40 & 4.35 & 45 & $\sqrt{ }$ \\
& $\sim 1.3$ & $5.12 \pm 0.20$ & 5.91 & 4.26 & 50 & \\
\hline \multirow{2}{*}{155} & $\sim 0.83$ & $4.30 \pm 0.17$ & 5.40 & 4.31 & 45 & $\sqrt{ }$ \\
& $\sim 1.0$ & $4.53 \pm 0.18$ & 5.98 & 4.20 & 50 & \\
\hline \multirow{2}{*}{165} & $\sim 0.35$ & $3.58 \pm 0.14$ & 5.02 & 4.34 & 35 & $\sqrt{ }$ \\
& $\sim 0.63$ & $3.75 \pm 0.15$ & 5.56 & 4.19 & 45 & \\
\hline \multirow{2}{*}{170} & $\sim 0.33$ & $3.25 \pm 0.15$ & 4.91 & 4.32 & 30 & $\sqrt{ }$ \\
\cline { 2 - 7 } & $\sim 0.38$ & $4.39 \pm 0.17$ & 5.57 & 4.26 & 35 & \\
\hline \hline
\end{tabular}

\section{Figure Captions}

Fig.1. Bottom mass for $\left.m_{t}=145 \mathrm{GeV}, a\right)$ as a function of $\lambda_{0, b}\left(M_{G U T}\right)$, and $\left.b\right)$ as a function of $r=\frac{\lambda_{0, b}}{\lambda_{0, t}}$ (see text for details).

Fig.2. Bottom mass for $m_{t}=170 \mathrm{GeV}$ as a function of $\tan \beta$.

Fig.3. Bottom mass for $m_{t}=145$ and $170 \mathrm{GeV}$. The shaded area is prevented due to unacceptable $\frac{m_{u}}{m_{c}}$ ratio. 


\section{References}

[1] S. Dimopoulos, L. Hall, and S. Raby, Phys. Rev. Lett. 68 (1992) 1984; ibid Ohio State preprint, DOE-ER-01545-567;

[2] G. Giudice, University of Texas preprint, UTTG-5-92, March, 1992;

[3] V. Barger, M.S. Berger, T. Han, and M. Zralek, Phys. Rev. Lett. 68 (1992) 3394; R.G. Roberts and G.G. Ross,Oxford preprint/92.

[4] S.Babu and Q. Shafi, Bartol preprint 1992(Phys.Lett.B to appear)

[5] H. Dreiner, G.K. Leontaris and N.D. Tracas,Oxford Preprint/92, National Technical University preprint NTUA 33/92, IOA-September/92.

[6] F.L. White et all, Ap.J.Lett. 396(1992)L13.

[7] GALLEX collaboration, Phys. Lett. B 285 (1992) 376, ibid 390.

[8] L. Wolfenstein, Phys. Rev. D 17 (1977) 2369; S.P.Mikheyev and A. Y. Smirnov, Nuov. Cim. C 9 (1986) 17.

[9] G.Gasser and H. Leutwyler, Phys.Rep.87(1982)77.

[10] Patricle Properties Data Book, Jule 1992. 